\title{
Some computations on the characteristic variety of a line arrangement
}

\section{Oscar Papini ${ }^{1}$ - Mario Salvetti ${ }^{1}$}

Dedicated to the memory of Ştefan Papadima

Received: 1 April 2019 / Revised: 28 November 2019 / Accepted: 17 July 2020 /

Published online: 17 August 2020

(c) The Author(s) 2020

\section{Abstract}

We find monodromy formulas for line arrangements that are fibered with respect to the projection from one point. We use them to find 0-dimensional translated components in the first characteristic variety of the arrangement $\mathcal{R}(2 n)$ determined by a regular $n$-polygon and its diagonals. We also find new 1-dimensional translated components which generalize the well-known case of the $B_{3}$-deleted arrangement.

Keywords Hyperplane arrangements - Characteristic varieties · Local cohomology

Mathematics Subject Classification $32 \mathrm{~S} 22 \cdot 55 \mathrm{~N} 25 \cdot 32 \mathrm{~S} 50$

\section{Introduction}

An abelian local system $\mathcal{L}_{\rho}$ on the complement $\mathcal{M}(\mathcal{A})$ of a hyperplane arrangement $\mathcal{A}$ in $\mathbb{C}^{N}$ is defined by choosing one non-zero complex number $\rho_{\ell}$ for each hyperplane $\ell \in$ $\mathcal{A}$. For a generic choice of parameters $\rho_{\ell}$, it is well known that homology concentrates in the top dimension $N$ (see e.g. [14]). The $i$-th characteristic variety of $\mathcal{A}$ is the subvariety

$$
V_{i}(\mathcal{A})=\left\{\left(\rho_{\ell}\right)_{\ell \in \mathcal{A}} \in\left(\mathbb{C}^{*}\right)^{r}: \operatorname{dim}\left(H_{i}\left(\mathcal{M}(\mathcal{A}) ; \mathcal{L}_{\rho}\right)\right)>0\right\}
$$

Partially supported by: progetto PRA “Geometria e Topologia delle Varietà" (2018), Università di Pisa; INdAM; MIUR.

\footnotetext{
$凶 \quad$ Mario Salvetti

salvetti@dm.unipi.it

Oscar Papini

oscar.papini@for.unipi.it

1 Department of Mathematics, University of Pisa, 56126 Pisa, Italy 
where $r=\# \mathcal{A}$. Broad literature on this subject is known, also in connection with the theory of resonance varieties and the study of the cohomology of the associated Milnor fiber (see e.g. [1,3-6,8-12,15,17-19,21,24,25,27,28]).

Probably the main problem is understanding if the characteristic varieties are combinatorially determined (see e.g. [16]). This is known to be true for their "homogeneous part", which corresponds to the resonance variety by the tangent cone theorem (see e.g. [7] for general references). Meanwhile the translated components of the characteristic variety are still not well understood; in particular, the geometric description which is known for the translated components of dimension at least 1 does not work in the same way for the 0-dimensional translated components (see also [2]).

For these reasons we think that it can be useful to produce more examples (interesting in themselves) such that the characteristic variety has some translated 0-dimensional essential component.

In this paper we outline a different approach, based on an (apparently new) elementary description of the characteristic variety. We consider here the case $N=2$. We find that the arrangement $\mathcal{R}(2 n)$ determined by a regular $n$-polygon and its diagonals produces the above mentioned phenomenon for $n \geqslant 5$, namely we find $\phi(n)$ translated 0-dimensional components in its characteristic variety (here $\phi$ is the Euler function). This fact was experimentally observed in [20] by using computer methods, up to $n=7$.

The arrangement $\mathcal{R}(2 n)$ belongs to the class of fibered arrangements: the projection through its "center" gives a fibration of the complement $\mathcal{M}(\mathcal{A})$ over $B=\mathbb{C} \backslash\{n-1$ points $\}$ with fiber $F=\mathbb{C} \backslash\{n$ points $\}$.

We obtain the above result by using algebraic complexes which compute the parallel transport and the monodromy of the first homology group of the fiber, which we think are interesting in themselves. For example, we deduce a restriction on essential components of the characteristic variety (Theorem 2.9) and a description of $V(\mathcal{A})$ $\left(=V_{1}(\mathcal{A})\right)$ as the set of points such that the transpose of the monodromy operators have a common eigenvector (Theorem 2.10).

For the arrangement $\mathcal{R}(2 n)$, we use the algebraic complexes constructed in Sect. 2 to make explicit computations. If $\omega_{n}$ is an $n$-th primitive root of 1 , we find that assigning to the edges of the polygon the parameter $\left(\omega_{n}\right)^{k}$ and to the diagonals the parameter $\left(\omega_{n}\right)^{k(n-2)}$, for $k=1, \ldots, n-1$, lowers the rank of the boundary operator in the algebraic complex computing $H_{0}\left(B ; H_{1}\left(F ; \mathcal{L}_{\mid F}\right)\right)$, therefore the corresponding point $P_{n, k}$ belongs to $V(\mathcal{A})$. In case $k$ and $n$ are coprime, we deduce that $P_{n, k}$ is an isolated point in the characteristic variety. Our argument works for $n \geqslant 5$ and uses the description (Theorem 2.10) of the characteristic variety and the remark that triple points give simple eigenvalues for the corresponding monodromy operators. We find that the shape of the common eigenvector imposes equalities among the eigenvalues of the monodromy operators that define a 0 -dimensional locus.

When $n$ and $k$ are not coprime, our argument can also be used. In this case the shape of the common eigenvector imposes less equalities (so, a higher dimensional locus in general). For $n=8$, we find (Theorem 3.7) a translated 1-dimensional component which contains the two points $P_{8,2}$ and $P_{8,6}$ (in contrast with [26, pp.45-46]; see Remark 3.6). One can easily see that such translated component cannot be obtained by using the method of [6, Section 5.5]; therefore our method seems indeed novel. This 
last construction generalizes to the case $n=4 m$ : we find an explicit 1-dimensional translated component containing $P_{n, m}$ and $P_{n, 3 m}$ (Theorem 3.12). When $n=4$, this gives another description of the 1-dimensional translated component found in [24]. The examples in Theorems 3.7 and 3.12 are the first examples of translated 1-dimensional components to be found after [24], not obtained by the method in [6] (see Remark 3.9).

We also remark that our approach can be generalized (with some modifications) to not necessarily fibered arrangements (we will return to this in future work).

\section{Algebraic complexes}

In this section we produce an algebraic complex computing local homology for a line arrangement. This complex seems particularly convenient in case of a fibered arrangement, in which case it is smaller than the one in [12,22].

Let $\mathcal{A}$ be an affine line arrangement; we assume that $\mathcal{A}$ is defined over $\mathbb{R}$ because formulas are easier to write, but all we say can be generalized to general complex arrangements. Let $\mathcal{M}(\mathcal{A})$ be its complement in $\mathbb{C}^{2}=\{(x, y): x, y \in \mathbb{C}\} ;$ let $\pi: \mathbb{C}^{2} \rightarrow$ $\mathbb{C}$ be the projection $\pi(x, y)=x$ onto the $x$-axis, and $\pi^{\prime}=\pi \mid \mathcal{N}(\mathcal{A}): \mathcal{N}(\mathcal{A}) \rightarrow \mathbb{C}$ its restriction. Let $\mathcal{S} \subset \mathbb{C}^{2}$ be the set of singular points of the arrangement, $\pi(\mathcal{S}) \subset \mathbb{C}$ its projection, and $B=\mathbb{C} \backslash \pi(\mathcal{S})$. Then

$$
\pi^{\prime \prime}=\pi_{\mid\left(\pi^{\prime}\right)^{-1}(B)}^{\prime}:\left(\pi^{\prime}\right)^{-1}(B) \rightarrow B
$$

is a fiber bundle with fibers $F_{x}:=\left(\pi^{\prime}\right)^{-1}(x), x \in B$. We divide the arrangement

$$
\mathcal{A}=\left\{\ell_{1}, \ldots, \ell_{n}, \ell_{1}^{\prime}, \ldots, \ell_{m}^{\prime}\right\}
$$

into horizontal and vertical lines, meaning that $\ell_{j}^{\prime}=\pi^{-1}(\xi)$ for some $\xi \in \pi(\mathcal{S})$, while $\pi\left(\ell_{i}\right)=\mathbb{C}$. Notice that if for all $\xi \in \pi(\mathcal{S})$ the vertical line $\pi^{-1}(\xi)$ is in $\mathcal{A}$, then $\mathcal{M}(\mathcal{A})=\left(\pi^{\prime}\right)^{-1}(B)$, so the whole complement $\mathcal{M}(\mathcal{A})$ is a fiber bundle over $B$. Let $\mathbb{C}_{x}=\pi^{-1}(x), x \in B$; then $F_{x}=\mathbb{C}_{x} \backslash\left\{y_{1}, \ldots, y_{n}\right\}$, where $y_{i}=\mathbb{C}_{x} \cap \ell_{i}$ for $i=1, \ldots, n$.

We fix a fiber $F_{0}=\left(\pi^{\prime \prime}\right)^{-1}\left(x_{0}\right), x_{0} \in B, x_{0} \in \mathbb{R}$; take a basepoint $P_{0} \equiv\left(x_{0}, y_{0}\right) \in$ $F_{0}$ with $\operatorname{Im}\left(y_{0}\right) \gg 0$, and elementary well-ordered generators $\alpha_{1}, \ldots, \alpha_{n}$ of $\pi_{1}\left(F_{0}\right)$, where $\alpha_{i}$ is constructed by using a path connecting $P_{0}$ to a small circle around $\ell_{i} \cap F_{0}$. The indices of the lines are taken according to the growing intersections with the real axis $\operatorname{Re}\left(\mathbb{C}_{x_{0}}\right)$.

We can make the same construction for any fiber $F_{x}, x \in B, x \in \mathbb{R}$, by using the basepoint $P_{0}^{(x)} \equiv\left(x, y_{0}\right)$ and generators $\alpha_{i}^{(x)}$; the indices here are locally computed according to the growing intersections of the lines with $\operatorname{Re}\left(\mathbb{C}_{x}\right)$.

We are interested in the local homology of $\mathcal{M}(\mathcal{A})$, where we consider the abelian local system defined by taking $\mathbb{C}$ as an $H_{1}(\mathcal{M}(\mathcal{A}) ; \mathbb{Z})$-module: the action is given by taking standard generators (small circles around the hyperplanes) into multiplication by non-zero numbers. So, such local systems correspond to $(n+m)$-tuples of parameters in $\left(\mathbb{C}^{*}\right)^{n+m}$. 
We call $s_{1}, \ldots, s_{n}$ and $t_{1}, \ldots, t_{m}$ the parameters corresponding to the lines $\ell_{1}, \ldots, \ell_{n}$ and $\ell_{1}^{\prime}, \ldots, \ell_{m}^{\prime}$, respectively. Denote by $\mathcal{L}=\mathcal{L}_{s, t}$ the corresponding local system. We also set $\mathcal{L}_{s}=\mathcal{L}_{F_{0}}$.

In this paper we will assume that $\mathcal{M}(\mathcal{A})$ fibers over $B$; it corresponds, as said before, to the case where the singular set $\mathcal{S}$ is contained in the union of vertical lines.

Lemma 2.1 We have

$$
H_{1}\left(\mathcal{M}(\mathcal{A}) ; \mathcal{L}_{s, t}\right)=H_{0}\left(B ; H_{1}\left(F_{0} ; \mathcal{L}_{s}\right)\right) \oplus H_{1}\left(B ; H_{0}\left(F_{0} ; \mathcal{L}_{s}\right)\right)
$$

where the second factor on the right vanishes if $\mathcal{L}_{s}$ is non-trivial (i.e., some $s_{i} \neq 1$ ).

Proof By standard constructions (see e.g. [13, Section 4.17]),

$$
H_{1}\left(\mathcal{M}(\mathcal{A}) ; \mathcal{L}_{s, t}\right)=H_{0}\left(B ; H_{1}\left(\left\{F_{x}\right\} ; \mathcal{L}_{s, t}\right)\right) \oplus H_{1}\left(B ; H_{0}\left(\left\{F_{x}\right\} ; \mathcal{L}_{s, t}\right)\right)
$$

This is (1) when we consider the homology groups $H_{*}\left(F_{0} ; \mathcal{L}_{s}\right) \cong H_{*}\left(F_{0} ; \mathcal{L}_{s, t}\right)$ as $\pi_{1}\left(B, x_{0}\right)$-modules and the well-known correspondence between local systems and representations of the fundamental group on one fixed fiber (see [23]). The 0-th local homology group is well known to vanish for non-trivial coefficients.

So we have to compute the term $H_{0}\left(B ; H_{1}\left(F_{0} ; \mathcal{L}_{s}\right)\right)$, where $H_{1}\left(F_{0} ; \mathcal{L}_{s}\right)$ is seen as a $\pi_{1}(B)$-module (actually, an $H_{1}(B ; \mathbb{Z})$-module); the precise form of the action will be included in Theorem 2.8 .

Lemma 2.2 If $\mathcal{L}_{s}$ is non-trivial, then $\operatorname{dim}\left(H_{1}\left(F_{0} ; \mathcal{L}_{s}\right)\right)=n-1$. As generators we can take the classes of

$$
\tilde{\alpha}_{i, i+1}=\left(1-s_{i+1}\right) \alpha_{i}-\left(1-s_{i}\right) \alpha_{i+1}, \quad i=1, \ldots, n-1,
$$

if no $s_{i}$ equals 1 .

Proof The fiber $F_{0}$ deformation retracts onto a wedge of $n 1$-spheres, given by $\bigcup_{i=1}^{n} \alpha_{i}$. Therefore by standard methods to compute the homology we are done.

To compute the monodromy on $H_{1}$, we need to understand the parallel transport

$$
\gamma\left(x, x^{\prime}\right)_{*}: H_{1}\left(F_{x} ; \mathcal{L}_{x}\right) \rightarrow H_{1}\left(F_{x^{\prime}} ; \mathcal{L}_{x^{\prime}}\right)
$$

where $\gamma\left(x, x^{\prime}\right)$ is a path in $B$ connecting $x$ with $x^{\prime}$ and $\mathcal{L}_{x}=\mathcal{L}_{\mid F_{x}}$. We compute the parallel transport for any points $x, x^{\prime} \in \mathbb{R} \backslash \pi(\mathcal{S})$, where $\mathbb{R}$ is the real axis of the first coordinate (recall that we are assuming $\pi(\mathcal{S}) \subset \mathbb{R}$ ). We denote by $\widetilde{\alpha}_{i, i+1}^{(x)}$, $i=1, \ldots, n-1$, the generators of $H_{1}\left(F_{x} ; \mathcal{L}_{x}\right)$, constructed as those in (2), by using the $\alpha_{i}^{(x)}$ 's. Formulas look better if we take as generators

$$
\alpha_{i, i+1}=\frac{\widetilde{\alpha}_{i, i+1}}{\left(1-s_{i}\right)\left(1-s_{i+1}\right)}=\frac{\alpha_{i}}{1-s_{i}}-\frac{\alpha_{i+1}}{1-s_{i+1}} .
$$


Of course, this requires each $s_{i} \neq 1$, but since we are interested in essential components of the characteristic variety (i.e., not contained in any coordinate tori $s_{i}=1$ ), this is not a serious restriction.

Given $x, x^{\prime} \in \mathbb{R} \backslash \pi(\mathcal{S})$, we consider a path $\gamma(t)=\gamma\left(x, x^{\prime}\right)(t), t \in[0,1]$, in $B$, connecting $x$ and $x^{\prime}$, and such that $\gamma \cap \mathbb{R}=\left\{x, x^{\prime}\right\}$ and $\operatorname{Im}(\gamma(t))\left(x-x^{\prime}\right) \geqslant 0$ for all $t \in[0,1]$ (i.e., $\gamma$ leaves the real axis on the left while traveling from $x$ to $x^{\prime}$ ). We denote by $\tau\left(x, x^{\prime}\right)$ the corresponding transport isomorphism (3).

Let $\sigma=\sigma\left(x, x^{\prime}\right)$ be the permutation of the indices $1, \ldots, n$ which is obtained as follows: the $i$-th line in the $x$-ordering (considering the growing intersections with $\left.\operatorname{Re}\left(\mathbb{C}_{x}\right)\right)$ is the $\sigma(i)$-th line in the $x^{\prime}$-ordering. For each $i=1, \ldots, n-1$ we set $\sigma(i, i+1)=+1$ or -1 depending on whether $\sigma(i+1)>\sigma(i)$ or $\sigma(i+1)<\sigma(i)$.

Theorem 2.3 (Parallel transport) We have

$$
\tau\left(x, x^{\prime}\right)\left(\alpha_{i, i+1}^{(x)}\right)=\sigma(i, i+1) \sum_{j=m(i, i+1)}^{M(i, i+1)-1} \prod_{\substack{k>i \\ \sigma(k) \leqslant j}} s_{k} \alpha_{j, j+1}^{\left(x^{\prime}\right)} .
$$

Here $m(i, i+1)=\min (\sigma(i), \sigma(i+1))$ and $M(i, i+1)=\max (\sigma(i), \sigma(i+1))$.

Proof The proof is a standard computation and it is obtained by the following steps. First, remark that any generator in (2) is obtained from Fox calculus from the commutator $\left[\alpha_{i}, \alpha_{i+1}\right]$ as

$$
\widetilde{\alpha}_{i, i+1}=\sum_{j=1}^{n} \varphi\left(\frac{\partial}{\partial \alpha_{j}}\left[\alpha_{i}, \alpha_{i+1}\right]\right) \alpha_{j}
$$

where $\varphi: \mathbb{Z}\left[\pi_{1}\left(F_{0}\right)\right] \rightarrow \mathbb{Z}\left[s_{1}^{ \pm 1}, \ldots, s_{n}^{ \pm 1}\right]$ is the valuation homomorphism taking $\alpha_{i}$ to $s_{i}$.

Next, we remark that while the path $\gamma$ turns around the projection of some singularity of a half-circle, the corresponding points in the vertical line make a half-twist, giving rise to a "local permutation" which takes a sequence of consecutive numbers to the opposite sequence (i.e., $k, \ldots, k+h$ goes to $k+h, \ldots, k$ ).

By induction on the number of points in $\pi(\mathcal{S})$ separating $x$ from $x^{\prime}$, we easily see that a generator $\alpha_{i}^{(x)}$ is taken by $\gamma$ to $P \alpha_{\sigma(i)}^{\left(x^{\prime}\right)} P^{-1}$, where $P$ is the product (in increasing order) of the $\alpha_{j}^{\left(x^{\prime}\right)}$ such that $j<\sigma(i)$ and $\sigma^{-1}(j)>i$. Then we apply Fox calculus as in (5) to the transform $\gamma_{*}\left(\left[\alpha_{i}^{(x)}, \alpha_{i+1}^{(x)}\right]\right)$ and we conclude.

The local monodromy around one point $p \in \pi(\mathcal{S})$ is obtained by taking a point $x \in \mathbb{R}$ very close to $p$ and transporting the fiber of $\pi^{\prime \prime}$ starting from $x$ around a circle centered at $p$. If $x^{\prime} \in \mathbb{R}$ is a point symmetric to $x$ with respect to $p$, the local monodromy $\mu_{x}$ is the automorphism of $H_{1}\left(F_{x} ; \mathcal{L}_{x}\right)$ which is obtained by composing $\tau\left(x^{\prime}, x\right) \circ \tau\left(x, x^{\prime}\right)$. The "local permutation" $\sigma\left(x, x^{\prime}\right)$ is determined by a partition $\Delta_{1}, \Delta_{2}, \ldots, \Delta_{h}$ of the set $\{1, \ldots, n\}$, where each $\Delta$ is composed of consecutive numbers $a, a+1, \ldots, a+r$ which are the indices (in the $x$-ordering) of the lines which intersect in a singular 
point $P$ on the vertical line $\mathbb{C}_{p}$ (so the multiplicity of $P$ as a singular point of $\mathcal{A}$ equals $\# \Delta+1)$.

Theorem 2.4 (Local monodromy) With the previous notations, the local monodromy around $p$ is given by

$$
\mu_{x}\left(\alpha_{i, i+1}^{(x)}\right)=\left\{\begin{array}{l}
\prod_{j \in \Delta} s_{j} \alpha_{i, i+1}^{(x)} \\
\alpha_{i, i+1}^{(x)}+\sum_{k=a}^{i-1}\left(1-s_{a} \cdots s_{k}\right) \alpha_{k, k+1}^{(x)} \\
\quad+\sum_{k=i+2}^{b} s_{i+1} \cdots s_{k-1}\left(1-s_{k} \cdots s_{b}\right) \alpha_{k-1, k}^{(x)}
\end{array}\right.
$$

where (a) occurs if $i$ and $i+1$ belong to the same block $\Delta$ of $\sigma\left(x, x^{\prime}\right)$ and (b) occurs if $i$ is the last element of the block $\{a, a+1, \ldots, i\}$ and $i+1$ is the first element of the next block $\{i+1, i+2, \ldots, b\}$.

Proof The proof directly follows from (4) and $\mu_{x}\left(\alpha_{i, i+1}^{(x)}\right)=\tau\left(x^{\prime}, x\right) \circ \tau\left(x, x^{\prime}\right)$.

As an immediate consequence of (6) we have

Corollary 2.5 Let $p \in \pi(\mathcal{S})$ and let $P_{1}, \ldots, P_{h}$ be the singular points of $\mathcal{A}$ in the vertical line $\mathbb{C}_{p}$, having multiplicities $m_{1}, \ldots, m_{h}$, respectively $\left(m_{1}+\cdots+m_{h}=\right.$ $n+h)$. Then the local monodromy $\mu_{x}$ has characteristic polynomial

$$
p_{x}(\lambda)=(\lambda-1)^{h-1} \prod_{i=1}^{h}\left(\lambda-\prod_{P_{i} \in \ell} s_{\ell}\right)^{m_{i}-2}
$$

and it is diagonalizable if all eigenvalues of the form $\prod s_{\ell}$ are different from 1 . Here we use $s_{\ell}$ to denote the parameter corresponding to the horizontal line $\ell$.

Now we consider the global monodromy $\mu: \pi_{1}\left(B, x_{0}\right) \rightarrow \operatorname{Aut}\left(H_{1}\left(F_{0} ; \mathcal{L}_{s}\right)\right)$. For each point $p \in \pi(\mathcal{S})$ we denote by $x_{p}, x_{p}^{\prime} \in \mathbb{R}$ two points close to $p$ and lying on opposite sides of $p$ in $\mathbb{R}$; assume that $x_{p}$ is the point lying in the segment $\left[p, x_{0}\right] \subset \mathbb{R}$.

The easiest way to compute monodromy is to take generators $\delta_{p}=\gamma\left(x_{0}, x_{p}^{\prime}\right)$ - $\gamma\left(x_{p}^{\prime}, x_{0}\right)$ for $\pi_{1}\left(B, x_{0}\right)$; such generator circles around all the projections of the singular points $p^{\prime} \in\left[p, x_{0}\right]$ counterclockwise. By using the parallel transport (4) we get

Theorem 2.6 (Global monodromy I) The global monodromy $\mu$ is determined by

$$
\begin{aligned}
& \mu\left(\left[\delta_{p}\right]\right)\left(\alpha_{i, i+1}\right)=\sum_{j=1}^{n-1} \mu_{j}^{i} \alpha_{j, j+1} \text { where } \\
& \mu_{j}^{i}=\sigma(i, i+1) \sum_{\substack{[k, k+1] \subset[\sigma(i), \sigma(i+1)] \\
[j, j+i] \subset\left[\sigma^{-1}(k), \sigma^{-1}(k+1)\right]}} \sigma^{-1}(k, k+1) \prod_{\substack{h \geqslant i+1 \\
\sigma(h) \leqslant k}} s_{h} \prod_{\substack{l \geqslant k+1 \\
\sigma^{-1}(l) \leqslant j}} s_{\sigma^{-1}(l)} .
\end{aligned}
$$


Here $[a, b]$ stands for the segment connecting the two points $a$ and $b$ in the real axis (of $\mathbb{C}_{x_{p}^{\prime}}$ and $\mathbb{C}_{x_{0}}$ ).

It is convenient to take elementary generators of $\pi_{1}\left(B, x_{0}\right)$ associated with $p$ : the path $\gamma_{p}$ is composed with the sequence

$$
\gamma_{p}=\gamma\left(x_{0}, x_{p}\right) \gamma\left(x_{p}, x_{p}^{\prime}\right) \gamma\left(x_{p}^{\prime}, x_{p}\right) \gamma\left(x_{p}, x_{0}\right)^{-1} \text {. }
$$

Clearly such paths give a well-ordered set of elementary generators of $\pi_{1}\left(B, x_{0}\right)$, so the global monodromy is determined by their images.

Theorem 2.7 (Global monodromy II) The global monodromy $\mu$ is determined by the maps

$$
\mu\left(\left[\gamma_{p}\right]\right)=\tau\left(x_{0}, x_{p}\right)^{-1} \mu_{x_{p}} \tau\left(x_{0}, x_{p}\right)
$$

where $\tau\left(x_{0}, x_{p}\right)$ is the parallel transport in (4) and $\mu_{x_{p}}$ is the local monodromy in (6).

It is also possible to write explicit formulas for (9): formulas (4) and (6) make possible to compute the images of the generators $\alpha_{i, i+1}$; we do not report such formulas here because we do not need their explicit form now. Notice also that (9) and (8) are related by

$$
\mu\left(\left[\gamma_{p_{i}}\right]\right)=\mu\left(\left[\delta_{p_{i-1}}\right]\right)^{-1} \mu\left(\left[\delta_{p_{i}}\right]\right)
$$

where the $p_{i}$ are ordered according to increasing distance from $x_{0}$. We now come back to the computation of $H_{0}\left(B ; H_{1}\left(F_{0} ; \mathcal{L}_{s}\right)\right)$ in $(1)$. The space $B$ deformation retracts onto a wedge of 1-spheres, actually onto the union $\bigcup_{p \in \pi(\mathcal{S})} \gamma_{p}$, where the unique 0 -cell is $x_{0}$. By standard methods which compute local system homology we obtain

Theorem 2.8 The vector space $H_{0}\left(B ; H_{1}\left(F_{0} ; \mathcal{L}_{s}\right)\right)$ is the cokernel of the map

$$
\partial_{1}: \bigoplus_{p \in \pi(\mathcal{S})} H_{1}\left(F_{0} ; \mathcal{L}_{s}\right)\left[\gamma_{p}\right] \rightarrow H_{1}\left(F_{0} ; \mathcal{L}_{S}\right)\left[x_{0}\right]
$$

defined as

$$
\partial_{1}\left(\alpha\left[\gamma_{p}\right]\right)=\left(t_{p} \cdot \mu\left(\left[\gamma_{p}\right]\right)(\alpha)-\alpha\right)\left[x_{0}\right]
$$

where we set here $t_{p}$ for the parameter associated with the vertical line $\mathbb{C}_{p} \in \mathcal{A}$.

Let $M_{p}$ be the matrix of $\mu\left(\left[\gamma_{p}\right]\right)$ in the given basis. Then the matrix $B$ of $\partial_{1}$ consists of $m$ square blocks $B_{p}=t_{p} M_{p}-\mathrm{Id}$, one for each $p \in \pi(\mathcal{S})$, of order $n-1$, so $B=\left[B_{1}, \ldots, B_{m}\right]$. It follows that the block $B_{p}$ has rank lower than $n-1$ iff $t_{p}$ coincides with the inverse of an eigenvalue of $\mu\left(\left[\gamma_{p}\right]\right)$. Therefore either $t_{p}=1$ or $t_{p} \prod_{P_{i} \in \ell} s_{\ell}=1$ for some singular point $P_{i} \in \mathbb{C}_{p}$ of order at least 3 (see (7)). We obtain the following corollary. 
Theorem 2.9 The essential components of the characteristic variety of $\mathcal{A}$ are contained in

$$
W(\mathcal{A})=\bigcap_{p \in \pi(\mathcal{S})} \prod_{\substack{P \in \mathbb{C}_{p} \\ m(P) \geqslant 3}}\left\{\prod_{\substack{\ell \text { s.t. } \\ P \in \ell}} \rho_{\ell}-1\right\} \subset\left(\mathbb{C}^{*}\right)^{n+m}
$$

where we write $\rho_{\ell}$ for the parameter associated with the line $\ell$ (in our previous notations it is of the shape $s_{i}$ for horizontal lines and $t_{j}$ for vertical lines).

As another immediate consequence we get the following description of the characteristic variety of $\mathcal{A}$.

Theorem 2.10 The essential part of the characteristic variety $V(\mathcal{A})$ coincides with the set of values $(s, t) \in\left(\mathbb{C}^{*}\right)^{n+m}$ such that the transpose of the monodromy operators $\mu\left(\left[\gamma_{p}\right]\right)(s), p \in \pi(\mathcal{S})$, have a common eigenvector relative to eigenvalues $t_{p}^{-1}, p \in$ $\pi(\mathcal{S})$.

Proof Indeed, a linear relation among the rows of the boundary matrix $B$ is given by a vector $v$ such that ${ }^{\mathrm{t}} v B_{p}={ }^{\mathrm{t}} v\left(t_{p} M_{p}-\mathrm{Id}\right)=0, p \in \pi(\mathcal{S})$.

\section{Some computations}

We consider here the arrangement $\mathcal{R}(2 n)$ which is projectively given by taking the lines spanned by the edges of a regular $n$-polygon, together with all its $n$ diagonals (see Figs. 1 and 3 for the case $n=6$ and 7, respectively). Notice that there is an $n$-tuple point $P$ which is the intersection of the diagonals. For odd $n$, on each diagonal we have, besides $P$, one double point at the intersection with the middle point of an edge, and $(n-1) / 2$ triple points. For even $n, n=2 k$, we have $k$ diagonals passing through two opposite vertices of the $n$-polygon, each of them containing $k$ triple points; and other $k$ diagonals passing through the middle points of two opposite sides of the $n$ polygon, each of them containing two double points and $k-1$ triple points. Of course, the projection from $P$ makes the complement $\mathcal{M}(\mathcal{A})$ a fiber bundle. We take one of the diagonals to infinity, so the projection $\pi: \mathbb{C}^{2} \rightarrow \mathbb{C}$ describes $\mathcal{M}(\mathcal{A})$ as a fiber bundle over $\mathbb{C} \backslash\{n-1$ points $\}$ with fiber $\mathbb{C} \backslash\{n$ points $\}$ (see Figs. 2 and 4 ).

Let $\ell_{1}^{\prime}=\left\{x=p_{1}\right\}, \ldots, \ell_{n-1}^{\prime}=\left\{x=p_{n-1}\right\}, p_{i} \in \mathbb{R}$, be the vertical lines, ordered according to $p_{1}>\cdots>p_{n-1}$. In the notations of the previous section, let $x_{0} \in \mathbb{R}$ be a basepoint in the $x$-coordinate: we choose $x_{0} \gg p_{1}$. Let also $\ell_{1}, \ldots, \ell_{n}$ be the horizontal lines, ordered according to increasing intersections with the real axis of $\mathbb{C}_{0}$. Fix also points $x_{i}=p_{i}+\epsilon, x_{i}^{\prime}=p_{i}-\epsilon$, with $i=1, \ldots, n-1$ and $0<\epsilon \ll 1$. We trivially have (see Figs. 2 and 4)

\section{Lemma 3.1}

(i) For odd $n$ the local permutation $\sigma_{i}=\sigma\left(x_{i}, x_{i}^{\prime}\right)$ is given by

- $\sigma_{i}=(1,2)(3,4) \cdots(n-2, n-1)(n)$ if $i$ is odd; 


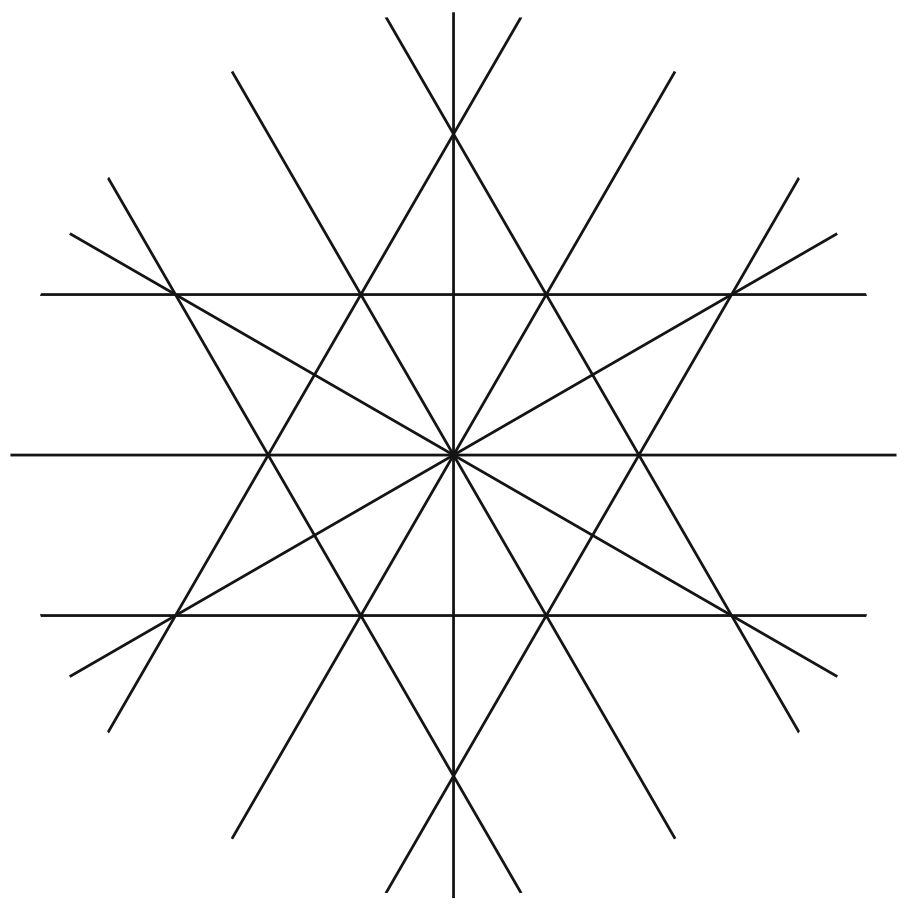

Fig. 1 The arrangement $\mathcal{R}(12)$ (projective picture)

- $\sigma_{i}=(1)(2,3)(4,5) \cdots(n-1, n)$ if $i$ is even.

(ii) For even $n$ the local permutation $\sigma_{i}=\sigma\left(x_{i}, x_{i}^{\prime}\right)$ is given by

- $\sigma_{i}=(1)(2,3) \cdots(n-2, n-1)(n)$ if $i$ is odd;

- $\sigma_{i}=(1,2)(3,4) \cdots(n-1, n)$ if $i$ is even.

Let $\omega_{n}$ be a primitive $n$-th root of the unity. We use results from the previous section to find explicit points in the characteristic variety.

Theorem 3.2 The $n$ points $P_{n, k} \in\left(\mathbb{C}^{*}\right)^{2 n-1}, k=0, \ldots, n-1$, whose coordinates are given by the $(s, t)$ with $s_{j}=\omega_{n}^{k}$ for $j=1, \ldots, n$ and $t_{j}=\left(\omega_{n}^{k}\right)^{n-2}$ for $j=$ $1, \ldots, n-1$, belong to the characteristic variety.

Proof The case $k=0$ is trivial since $P_{n, 0}=(1, \ldots, 1)(2 n-1$ entries). So assume $k>0$. Let us consider the row vector

$$
v_{n}=\left[(-1)^{n-1} \frac{\omega^{n-1}-1}{\omega-1}, \ldots,(-1)^{j} \frac{\omega^{j}-1}{\omega-1}, \ldots,-1\right] \in \mathbb{C}^{n-1}
$$

where we set for brevity $\omega=\omega_{n}^{k}$. We prove that $v_{n}$ is an eigenvector for the transpose operator ${ }^{\mathrm{t}} \partial_{1}\left(\left[\gamma_{p}\right]\right)$ in (11) for all $\gamma_{p}$ when the parameters take the above values. This shows that such parameters lower the rank of $\partial_{1}$ in (10), so the corresponding point belongs to the characteristic variety. 


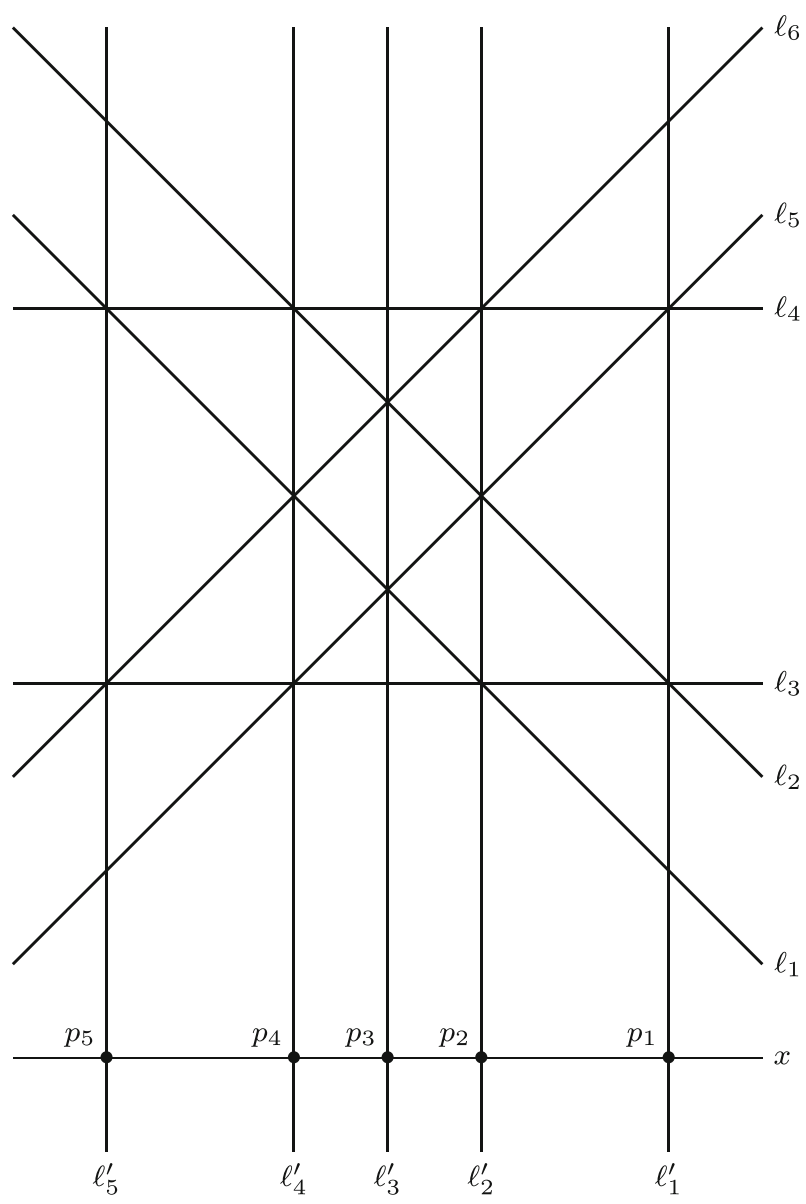

Fig. 2 The arrangement $\mathcal{R}(12)$ (affine picture) projected onto the $x$-axis

Notice that by Lemma 3.1 the local monodromy around $p_{i}$ coincides with the local monodromy $M_{1}$ around $p_{1}$ for odd $i$ or with the local monodromy $M_{2}$ around $p_{2}$ for even $i$. Therefore by the expression (9) for the monodromy it is sufficient to prove that $v_{n}$ is an eigenvector for ${ }^{\mathrm{t}} M_{1}$ and ${ }^{\mathrm{t}} M_{2}$ with parameters $s_{i}=\omega$, with respect to the eigenvalue $\omega^{n-2}$, and that both $\tau\left(x_{1}, x_{2}\right)^{-1}$ and $\tau\left(x_{2}, x_{3}\right)^{-1}$ take $v_{n}$ into an eigenvector of ${ }^{\mathrm{t}} M_{2},{ }^{\mathrm{t}} M_{1}$ respectively.

The first assertion is easily proven by looking at formulas (6). With the given parameters, there are as many zero columns in $t_{i} M_{i}-\mathrm{Id}$ as triple points. So, for odd $n$ there are $(n-1) / 2$ non-zero columns, at even position for $t_{1} M_{1}-$ Id, at odd position for $t_{2} M_{2}-\mathrm{Id}$; for even $n$, there are $n / 2$ non-zero columns at odd position for $t_{1} M_{1}-\mathrm{Id}$ and $(n-2) / 2$ non-zero columns at even position for $t_{2} M_{2}-$ Id. The non-zero $j$-th column has entries $\omega^{n-2}-1$ in position $(j, j)$, preceded (if it is not the first entry of the column) by $\omega^{n-2}(1-\omega)$, and followed (if it is not the last entry of the column) 


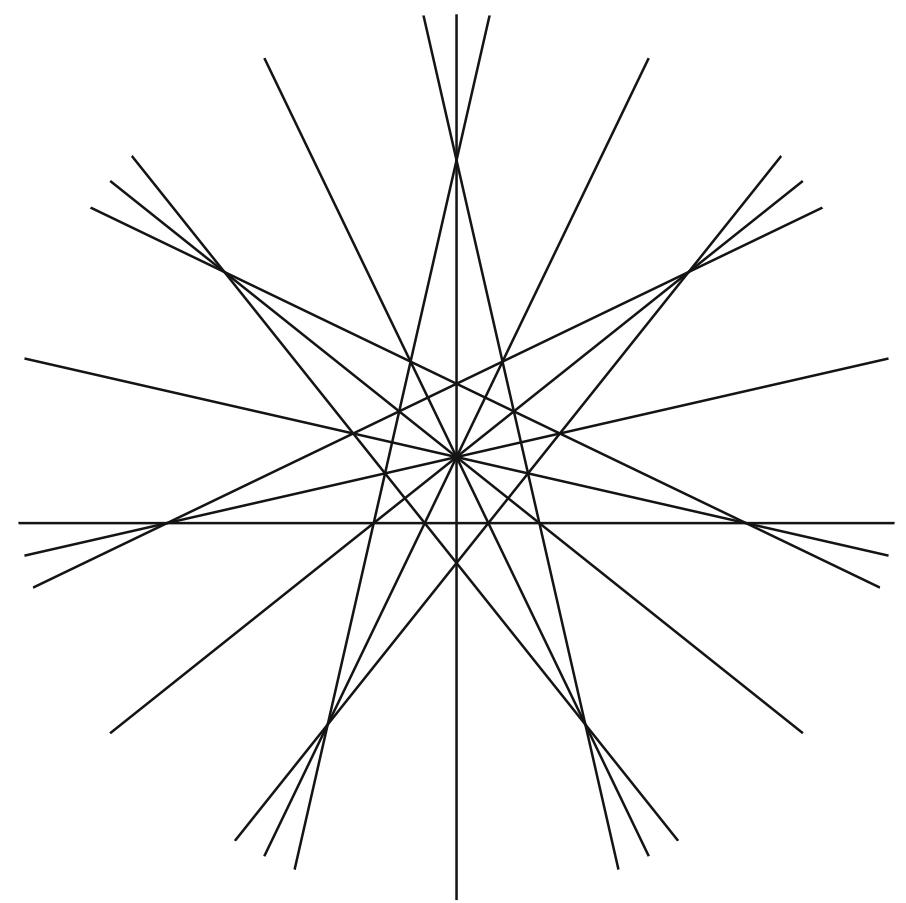

Fig. 3 The arrangement $\mathcal{R}(14)$ (projective picture)

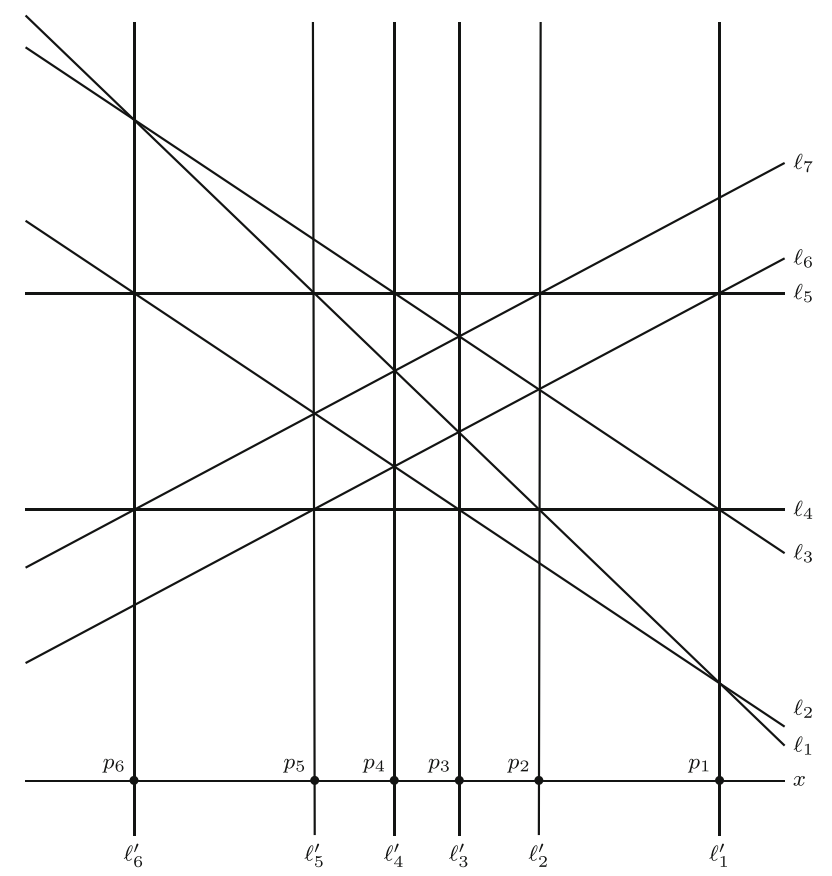

Fig. 4 The arrangement $\mathcal{R}(14)$ (affine picture) projected onto the $x$-axis 
by $\omega^{n-1}-1$; all other entries of the column vanish. Then it is easy to check that multiplying on the left by $v_{n}$ gives zero.

The form of $T=\tau\left(x_{1}, x_{2}\right)^{-1}$ and $T^{\prime}=\tau\left(x_{2}, x_{3}\right)^{-1}$ (with the given parameters) is derived from formula (4) and from Lemma 3.1. In case $n$ is odd one obtains:

- for odd $j, T_{j, j}=-\omega^{-1}$ and $T_{i, j}=0$ for $i \neq j$;

- for even $j, T_{j-1, j}=\omega^{-1}, T_{j, j}=T_{j+1, j}=1$, and $T_{i, j}=0$ for all other $i$ 's.

The shape of $T^{\prime}$ is completely analogous; it is enough to exchange the roles of even and odd $j$. For $n$ even, the shape is the same exchanging $T$ and $T^{\prime}$. Then it is easy to check directly that

$$
v_{n} \cdot T=v_{n} \cdot T^{\prime}=-\omega^{-1} v_{n}
$$

and we conclude.

Remark 3.3 By symmetry, there is an obvious action of the cyclic group $C_{n}$ generated by $\sigma_{n}=(0,1, \ldots, n-1)$ onto the characteristic variety. Consider the edges of the $n$-polygon (in the projective picture) cyclically ordered as $\ell_{0}, \ldots, \ell_{n-1}$. Let us cyclically order also the diagonals $\ell_{0}^{\prime}, \ldots \ell_{n-1}^{\prime}$, starting from the diagonal $\ell_{0}^{\prime}$ which is orthogonal to $\ell_{0}$. Take a new parameter $t_{n-1}$ for the line at infinity, with the condition that $\prod_{i=0}^{n-1} s_{i} \prod_{j=0}^{n-1} t_{j}=1$. Then the action of $\sigma_{n}$ on some point $\left(s_{0}, \ldots, s_{n-1}, t_{0}, \ldots\right.$, $\left.t_{n-1}\right) \in\left(\mathbb{C}^{*}\right)^{2 n}$ in $V$ is the point $\left(s_{\sigma_{n}(0)}, \ldots, s_{\sigma_{n}(n-1)}, t_{\sigma_{n}^{2}(0)}, \ldots, t_{\sigma_{n}^{2}(n-1)}\right)$ which also belongs to $V$. Notice that the points $P_{n, k}$ are fixed by this action.

With this labeling the double points are given by the intersection of the lines with indices $\left(i,(2 i)^{\prime}\right)$ and the triple points are given by the intersections of the lines with indices $\left(i, j,(i+j)^{\prime}\right)$ (taking indices $\left.\bmod n\right)$.

The next theorem needs a lemma.

Lemma 3.4 Let $n \geqslant 5$ and let $\omega_{n}$ be a primitive $n$-th root of 1 .

(i) Let $R_{S}=\mathbb{C}\left[s_{0}^{ \pm 1}, \ldots, s_{n-1}^{ \pm 1}\right]$ be the ring of Laurent polynomials in the variables $s_{0}, \ldots s_{n-1}$. The ideal

$$
I=\left(s_{i} s_{j}=s_{l} s_{m}: i+j \equiv l+m(\bmod n), i \neq j, l \neq m\right)
$$

is 1-dimensional with $n$ irreducible components

$$
I_{h}=\left\{s_{i}=\left(\omega_{n}\right)^{h i} s_{0}: i=1, \ldots, n-1\right\}, \quad h=0, \ldots, n-1 .
$$

(ii) Let $R_{s, t}=\mathbb{C}\left[s_{0}^{ \pm 1}, \ldots, s_{n-1}^{ \pm 1}, t_{0}^{ \pm 1}, \ldots, t_{n-1}^{ \pm 1}\right]$ be the ring of Laurent polynomials in $s_{i}, t_{j}, i, j=0, \ldots, n-1$. Let $J$ be the ideal

$$
J=\left(t_{i+j} s_{i} s_{j}=1, \prod_{i=0}^{n-1} s_{i} \prod_{i=0}^{n-1} t_{i}=1\right)
$$


where we take all possible pairs $(i, j)$ with $i \neq j$, and the indices are $\bmod n$. Then

$J$ is 0-dimensional and defines the $n^{2}$ points

$$
P_{n, h, k}=\left\{s_{i}=\left(\omega_{n}\right)^{h i+k}, t_{i}=\left(s_{0} s_{i}\right)^{-1}, i=0, \ldots, n-1\right\}, h, k=0, \ldots, n-1 .
$$

Proof (i) At first we prove the relations

$$
s_{i}^{2}=s_{j} s_{k} \text { for all } i, j, k \text { with } 2 i \equiv j+k(\bmod n) .
$$

By multiplying the two relations $(n>3)$

$$
s_{0} s_{1}=s_{2} s_{n-1} \quad \text { and } \quad s_{0} s_{n-1}=s_{1} s_{n-2}
$$

we deduce $s_{0}^{2}=s_{2} s_{n-2}$. Therefore (13) follows for $n>4$. So now we have all the relations of the form

$$
s_{i} s_{j}=s_{l} s_{m} \text { for all } i, j, l, m \text { with } i+j \equiv l+m(\bmod n)
$$

Then one easily deduces for recurrence the relations

$$
s_{a_{1}} \cdots s_{a_{k}}=s_{b_{1}} \cdots s_{b_{k}}
$$

each time $\sum a_{j}=\sum b_{j}(\bmod n)$. In particular, we have $s_{0}^{n}=s_{i}^{n}$ for all $i=1, \ldots, n-1$. Then $s_{1}=\left(\omega_{n}\right)^{h} s_{0}$ for some $h$. From $s_{i} s_{0}=s_{1} s_{i-1}$ it follows by induction that $s_{i}=\left(\omega_{n}\right)^{h i} s_{0}$ which proves (i).

For (ii), notice that the relations defining $I$ follow from those which define $J$, so we have again $s_{i}=\left(\omega_{n}\right)^{h i} s_{0}, i=0, \ldots, n-1$, for some $h$ in $0, \ldots, n-1$. Now using $t_{i}=\left(s_{0} s_{i}\right)^{-1}$ the last relation gives $\prod s_{i} \prod\left(s_{0} s_{i}\right)^{-1}=\left(s_{0}\right)^{-n}=1$. Therefore $s_{0}=\omega_{n}^{k}$ for some $k$ in $0, \ldots, n-1$.

In conclusion, we show

Theorem 3.5 The $\phi(n)$ points $P_{n, k}$ with $(n, k)=1$ of Theorem 3.2 are isolated points of the characteristic variety $V(\mathcal{A})$.

Proof We divide the proof into several steps.

I. We already know from Theorem 3.2 that $\operatorname{rk}\left(\partial_{1}\left(P_{n, k}\right)\right)<n-1, k=0, \ldots, n-1$. We directly check that $\operatorname{rk}\left(\partial_{1}\left(P_{n, k}\right)\right)=n-2$ if $(n, k)=1$.

Indeed, the $(n-1) \times 2(n-1)$-submatrix of $\partial_{1}$ which corresponds to the local monodromy around the first two points $p_{1}, p_{2}$ is already of rank $n-2$. This follows from the explicit description of the matrices $B_{i}:=t_{i} M_{i}-\mathrm{Id}, i=1$, 2, given in Theorem 3.2. By taking a basepoint between the two points $p_{1}$ and $p_{2}$ (which amounts to simultaneously conjugate the blocks of the boundary matrix), the two blocks $B_{1}$ and $B_{2}$ are submatrices of the corresponding boundary $\partial_{1}$. Reordering the $n-1$ non-zero columns of $B_{1}$ and $B_{2}$ gives a tridiagonal matrix of order $n-1$ which has clearly rank $n-2$. 
Therefore, $\operatorname{rk}\left(\partial_{1}\left(P^{\prime}\right)\right) \geqslant n-2$ if $P^{\prime}$ is a point close to $P_{n, k}$. We have to show that $\operatorname{rk}\left(\partial_{1}\left(P^{\prime}\right)\right)=n-1$ if $P^{\prime} \neq P_{n, k}$ and $P^{\prime}$ is close to $P_{n, k}$.

II. It follows from the previous point that the left kernel of $\partial_{1}$ is of dimension 1 in the given points, so it is spanned by the vector $v_{n}$ of Theorem 3.2. Notice that, under the hypothesis $(n, k)=1$, all the coordinates of $v_{n}$ are different from 0 . It implies that any $n-2$ rows of $\partial_{1}$ are linearly independent, and this must remain true in a neighborhood of $P_{n, k}$.

III. Recall from Theorem 2.10 that the vector $v_{n}$ is a common eigenvector for the transposed monodromy operators. Let us write the explicit form of the first block $t_{1}{ }^{\mathrm{t}} M_{1}-$ Id of the boundary operator for $n=6$ and 7 :

- for $n=6$,

$$
t_{1}{ }^{\mathrm{t}} M_{1}-\mathrm{Id}=\left[\begin{array}{ccccc}
t_{1}-1 & t_{1} s_{2}\left(1-s_{3}\right) & 0 & 0 & 0 \\
0 & t_{1} s_{2} s_{3}-1 & 0 & 0 & 0 \\
0 & t_{1}\left(1-s_{2}\right) & t_{1}-1 & t_{1} s_{4}\left(1-s_{5}\right) & 0 \\
0 & 0 & 0 & t_{1} s_{4} s_{5}-1 & 0 \\
0 & 0 & 0 & t_{1}\left(1-s_{4}\right) & t_{1}-1
\end{array}\right]
$$

- for $n=7$,

$$
t_{1}{ }^{\mathrm{t}} M_{1}-\mathrm{Id}=\left[\begin{array}{cccccc}
t_{1} s_{1} s_{2}-1 & 0 & 0 & 0 & 0 & 0 \\
t_{1}\left(1-s_{1}\right) & t_{1}-1 & t_{1} s_{3}\left(1-s_{4}\right) & 0 & 0 & 0 \\
0 & 0 & t_{1} s_{3} s_{4}-1 & 0 & 0 & 0 \\
0 & 0 & t_{1}\left(1-s_{3}\right) & t_{1}-1 & t_{1} s_{5}\left(1-s_{6}\right) & 0 \\
0 & 0 & 0 & 0 & t_{1} s_{5} s_{6}-1 & 0 \\
0 & 0 & 0 & 0 & t_{1}\left(1-s_{5}\right) & t_{1}-1
\end{array}\right]
$$

Having $\left(t_{1}{ }^{\mathrm{t}} M_{1}-\mathrm{Id}\right) v_{n}=0$ and all components of $v_{n}$ different from 0 clearly gives

$$
\begin{aligned}
& t_{1} s_{2} s_{3}=t_{1} s_{4} s_{5}=1 \text { for } n=6, \quad \text { and } \\
& t_{1} s_{1} s_{2}=t_{1} s_{3} s_{4}=t_{1} s_{5} s_{6}=1 \text { for } n=7 .
\end{aligned}
$$

These conditions are equivalent to $t_{1} s_{i} s_{j}=1$ each time $\ell_{1}^{\prime}, \ell_{i}, \ell_{j}$ form a triple point. Of course, we have the same conditions for all $n$.

IV. By symmetry, or changing coordinates by taking a point $x$ close to the vertical line $\ell_{i}^{\prime}$ as basepoint, we obtain similar conditions for any vertical line.

Remark also that we can take to infinity any other line passing through the center of the $n$-polygon, obtaining similar conditions for the line $\ell_{n}^{\prime}$ with given parameter $t_{n}$. Recall that in the projective situation, the product of all $s$ and $t$ equals 1 .

V. It follows that $P_{n, k}$ is contained in the zero locus of the ideal (with obvious notation)

$$
I=\left(t_{\ell} s_{\ell^{\prime}} s_{\ell^{\prime \prime}}=1: \ell \cap \ell^{\prime} \cap \ell^{\prime \prime} \text { is a triple point of } \mathcal{A}\right) \cap\left(\prod s_{i} \prod t_{j}=1\right)
$$


Now notice that any point $P^{\prime}$ very close to $P_{n, k}$ either does not belong to the characteristic variety or it corresponds to a common eigenvector $v_{n}^{\prime}$ still having non-vanishing components, so defining the same conditions. Therefore in a neighborhood of $P_{n, k}$ the characteristic variety is contained in the zero locus of (14).

By Lemma 3.4 the ideal $I$ is zero-dimensional (for $n \geqslant 5$ ), so we are done.

Remark 3.6 In [26, pp. 45-46] the case $n=8$ is considered and the authors claim that the six points $P_{8, k}$ for $k=1, \ldots, 7, k \neq 4$, are isolated points in the characteristic variety. The argument used in Theorem 3.5 works for $(n, k)=1$; nevertheless, it can be refined in the following way. For $n=8$ and $k=2,6$, the eigenvector $v_{8}$ in (12) has a unique zero component in the fourth coordinate:

$$
\begin{aligned}
& v_{8,2}=\left[-\omega^{2}, \omega^{2}+1,-1,0,-\omega^{2}, \omega^{2}+1,-1\right], \\
& v_{8,6}=\left[\omega^{2},-\omega^{2}+1,-1,0, \omega^{2},-\omega^{2}+1,-1\right]
\end{aligned}
$$

(where $\omega$ is a primitive 8-th root of the unity), and those are eigenvectors for both local monodromies ${ }^{t} M_{1}$ and ${ }^{t} M_{2}$. As in the proof of Theorem 3.5 we look at the explicit form of the local monodromy around the first two lines, which gives the two blocks in the boundary $\partial_{1}$ :

$$
\begin{aligned}
& t_{1}{ }^{\mathrm{t}} M_{1}-\mathrm{Id} \\
& =\left[\begin{array}{ccccccc}
t_{1}-1 & t_{1} s_{2}\left(1-s_{3}\right) & 0 & 0 & 0 & 0 & 0 \\
0 & t_{1} s_{2} s_{3}-1 & 0 & 0 & 0 & 0 & 0 \\
0 & t_{1}\left(1-s_{2}\right) & t_{1}-1 & t_{1} s_{4}\left(1-s_{5}\right) & 0 & 0 & 0 \\
0 & 0 & 0 & t_{1} s_{4} s_{5}-1 & 0 & 0 & 0 \\
0 & 0 & 0 & t_{1}\left(1-s_{4}\right) & t_{1}-1 & t_{1} s_{6}\left(1-s_{7}\right) & 0 \\
0 & 0 & 0 & 0 & 0 & t_{1} s_{6} s_{7}-1 & 0 \\
0 & 0 & 0 & 0 & 0 & t_{1}\left(1-s_{6}\right) & t_{1}-1
\end{array}\right], \\
& t_{2}{ }^{\mathrm{t}} M_{2}-\mathrm{Id} \\
& =
\end{aligned}
$$

The non-zero positions of $v_{8}$ give the equalities

$$
t_{1} s_{2} s_{3}=t_{1} s_{6} s_{7}=1
$$

from the first block, and

$$
t_{2} s_{1} s_{2}=t_{2} s_{3} s_{4}=t_{2} s_{5} s_{6}=t_{2} s_{6} s_{7}=1
$$

from the second block, which must hold in a neighborhood of $P_{8,2}$. 
Notice that the first vertical line corresponds in the projective picture to a line $\ell_{0}^{\prime}$ (notations of Remark 3.3) passing through the center and cutting two opposite edges of the octagon in the middle point. The second vertical line corresponds to a diagonal $\ell_{1}^{\prime}$ passing through two opposite vertices of the octagon. Among all triple points contained in these lines, the unique triple point which we are not considering in the equations (15) and (16) corresponds (in the projective picture) to that formed by $\ell_{0}^{\prime}$ and the two edges of the octagon which are "parallel" to $\ell_{0}^{\prime}$ (and parallel themselves).

By symmetry (or changing the coordinate system) we obtain analogous equations for all diagonals. That is, in a neighborhood of $P_{8,2}$ (and of $P_{8,6}$ ), the characteristic variety is contained in the zero locus of the ideal $I^{\prime}$ which is obtained from the ideal $I$ in (14) by deleting the four equations which correspond to the triple points determined by the four pairs of opposite edges of the octagon.

By making explicit computations we obtain that the ideal $I^{\prime}$ is 1-dimensional, and we finally get

Theorem 3.7 The characteristic variety of the arrangement $\mathcal{R}(16)$ contains a 1 dimensional translated component with the following parametrization:

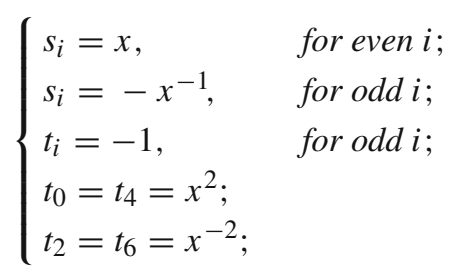

where $x$ is a parameter in $\mathbb{C}^{*}$. Such component contains the two points $P_{8,2}$ and $P_{8,6}$ (which are therefore not isolated).

Proof Once we have the explicit form (17) it is just a direct computation to see that it defines a component which is contained in the characteristic variety: we used both the expression of the matrix boundary $\partial_{1}$ of Theorem 2.8 and also the description of the boundary in [12] (of course both of them give that (17) is contained in the characteristic variety).

It is also possible to give a proof which parallels the one in Theorem 3.2. In particular, we find an explicit common eigenvector for the transposed monodromy operators (Theorem 2.10), which is given (in terms of the parameter $x$ ) by

$$
w_{8}(x)=[1, x-1,-x, 0,1, x-1,-x]
$$

It is immediate to see that $P_{8,2}$ and $P_{8,6}$ are contained in (17) (with parameters $x=\omega^{2}$, $x=\omega^{6}$, respectively); in fact, notice that vectors $v_{8,2}$ and $v_{8,6}$ above are obtained as $v_{8,2}=-\omega^{2} w_{8}\left(\omega^{2}\right)$ and $v_{8,6}=-\omega^{6} w_{8}\left(\omega^{6}\right)$.

As we have already remarked, in a neighborhood of these points the characteristic variety is 1-dimensional; therefore the component (17) is isolated. 
Remark 3.8 Case $n=4$, which corresponds to the $B_{3}$-deleted arrangement considered in [24], can also be included in our method. If we consider any of the points $P_{n, k}$, $k=1,2,3$, and we repeat the same construction as in Theorem 3.5, we obtain the same ideal $I$ in (14), which is in this case 1-dimensional. In fact, the zero locus of $I$ has four irreducible 1-dimensional components: one of these has the same shape as (17), being parametrized as

$$
\left\{\left(x,-x^{-1}, x,-x^{-1}, x^{2},-1, x^{-2},-1\right): x \in \mathbb{C}^{*}\right\}
$$

and it is the translated component appearing in [24]. It contains the two points $P_{4,1}$ and $P_{4,3}$. The other three components of the zero locus of $I$ do not belong to the characteristic variety. ( $P_{4,2}$ belongs to a different component of the zero locus of $I$, and that component is not fully contained in the characteristic variety.)

Remark 3.9 In [6, Proposition 5.6] the authors explain a method to find translated, 1-dimensional components in terms of pointed multinets, which are a special case of multinets. The $B_{3}$-deleted arrangement is an example of an arrangement whose 1-dimensional translated component is obtained with this method.

One more example is given in [20, Chapter 5], where the arrangement $Q(4)$ is given by ten projective lines with the following (affine) equation:

$$
(x-2)(x-1)(x+1)(x+2)(y-2)(y-1)(y+1)(y+2)(x+y)(x-y)=0 .
$$

If we give all the ten lines weight 1 and add the infinity line $\ell_{\infty}$ with weight 2 , we obtain an arrangement of eleven lines which supports a pointed multinet, with special line $\ell_{\infty}$. So $2(4)$ verifies the criterion in [6].

In case of the arrangement $\mathcal{R}(16)$ of Theorem 3.7, such method would apply if one could find a bigger arrangement $\mathcal{A}$ with 17 lines, containing $\mathcal{R}(16)$, which supports a pointed multinet. It is just an exercise to prove the following

Proposition 3.10 No arrangement $\mathcal{A}$ of 17 lines containing $\mathcal{R}(16)$ supports a multinet.

It follows that our example in Theorem 3.7 cannot be obtained by the method in [6].

Remark 3.11 Some other cases where $(n, k) \neq 1$ are known. For example, in case $n=9, k=3$, the point $P_{9,3}$ is contained in a 2-dimensional component coming from a multinet (see [11]).

The argument outlined in Remark 3.6 can be extended to any case $n=4 m$ to produce a 1-dimensional translated component, generalizing (19), (17).

Theorem 3.12 When $n=4 m$, the characteristic variety $V(\mathcal{A})$ contains a 1-dimensional translated component parametrized as (the labelings are as in Remark 3.3)

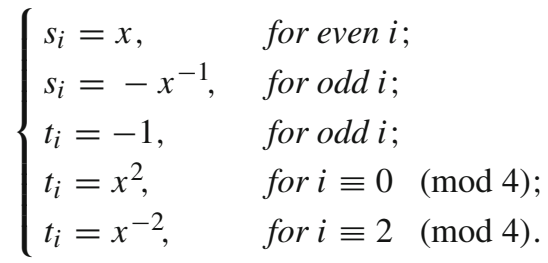


Proof We outline the proof of the theorem, which parallels that of Theorem 3.7.

As in Theorem 3.2, by using the explicit form of the boundary one verifies that the points in (20) lower the rank of the boundary matrix, so the component is contained in $V(\mathcal{A})$. We find a common eigenvector of the transposed monodromy operators, which generalizes (18),

$$
w_{n}(x)=[1, x-1,-x, 0,1, x-1,-x, 0, \ldots, 1, x-1,-x, 0,1, x-1,-x]
$$

(the triple $[1, x-1,-x]$ repeats $m$ times, separated by a 0 ).

Now consider the point $P_{n, m}$, which is contained in (20) (with $x=\omega^{m}$ ); a similar argument holds by using $P_{n, 3 m}$ instead. As in the proof of Theorem 3.7, for this point the vector $v_{n}$ in (12) is given by $v_{n}=-\omega^{m} w_{n}\left(\omega^{m}\right)$, where $\omega$ is a primitive $n$-th root of the unity. Therefore $v_{n}$ has $4 m-1$ entries which vanish exactly in the positions multiple of 4. The argument of Theorem 3.5 (or of Theorem 3.7) produces an ideal $I^{\prime}$ which is obtained from $I$ by deleting, for each even $i$ among $0, \ldots, 4 m-1$, the $m-1$ equations $t_{i} s_{(i / 2)+2 j} s_{(i / 2)-2 j}=1$, for $j=1, \ldots, m-1$ (indices are as in Remark 3.3 and are taken $\bmod n)$. One sees that the zero locus $Z\left(I^{\prime}\right)$ is of dimension 1 and (20) is contained in $Z\left(I^{\prime}\right)$ as an irreducible component which contains $P_{n, m}$ and $P_{n, 3 m}$.

Then we conclude that such component is not contained in a higher dimensional one by the same argument as used in Theorem 3.5 (and Theorem 3.7): points of the characteristic variety which are close to $P_{n, m}$ impose the same conditions, so they are contained in $Z\left(I^{\prime}\right)$.

Remark 3.13 Analogous computations can be done for other points $P_{n, k}$ where the number and the positions of the zero entries of the common eigenvector tell us what ideal to take. For example, we made some computations finding further isolated points in $V(\mathcal{A})$ for $n=12$. Details and further applications will be provided later.

Acknowledgements Open access funding provided by Università di Pisa within the CRUI-CARE Agreement.

Open Access This article is licensed under a Creative Commons Attribution 4.0 International License, which permits use, sharing, adaptation, distribution and reproduction in any medium or format, as long as you give appropriate credit to the original author(s) and the source, provide a link to the Creative Commons licence, and indicate if changes were made. The images or other third party material in this article are included in the article's Creative Commons licence, unless indicated otherwise in a credit line to the material. If material is not included in the article's Creative Commons licence and your intended use is not permitted by statutory regulation or exceeds the permitted use, you will need to obtain permission directly from the copyright holder. To view a copy of this licence, visit http://creativecommons.org/licenses/by/4.0/.

\section{References}

1. Arapura, D.: Geometry of cohomology support loci for local systems I. J. Algebraic Geom. 6(3), 563-597 (1997)

2. Artal Bartolo, E., Cogolludo-Agustín, J.I., Matei, D.: Characteristic varieties of quasi-projective manifolds and orbifolds. Geom. Topol. 17(1), 273-309 (2013)

3. Callegaro, F.: The homology of the Milnor fiber for classical braid groups. Algebr. Geom. Topol. 6, 1903-1923 (2006)

4. Cohen, D.C., Orlik, P.: Arrangements and local systems. Math. Res. Lett. 7(2-3), 299-316 (2000) 
5. Cohen, D.C., Suciu, A.I.: Characteristic varieties of arrangements. Math. Proc. Cambridge Philos. Soc. 127(1), 33-53 (1999)

6. Denham, G., Suciu, A.I.: Multinets, parallel connections, and Milnor fibrations of arrangements. Proc. London Math. Soc. 108(6), 1435-1470 (2014)

7. Dimca, A.: Hyperplane Arrangements. Universitext. Springer, Cham (2017)

8. Dimca, A., Papadima, Ş.: Finite Galois covers, cohomology jump loci, formality properties, and multinets. Ann. Sc. Norm. Super. Pisa Cl. Sci. 10(2), 253-268 (2011)

9. Dimca, A., Papadima, Ş., Suciu, A.I.: Topology and geometry of cohomology jump loci. Duke Math. J. 148(3), 405-457 (2009)

10. Falk, M.J.: Arrangements and cohomology. Ann. Combin. 1(2), 135-157 (1997)

11. Falk, M.J., Yuzvinsky, S.: Multinets, resonance varieties, and pencils of plane curves. Compositio Math. 143(4), 1069-1088 (2007)

12. Gaiffi, G., Salvetti, M.: The Morse complex of a line arrangement. J. Algebra 321(1), 316-337 (2007)

13. Godement, R.: Topologie algébrique et théorie des faisceaux. Actualités Sci. Ind. No. 1252. Publ. Math. Univ. Strasbourg. No. 13. Hermann, Paris (1958)

14. Kohno, T.: Homology of a local system on the complement of hyperplanes. Proc. Japan Acad. Ser. A Math. Sci. 62(4), 144-147 (1986)

15. Libgober, A.: Characteristic varieties of algebraic curves. In: Ciliberto, C., et al. (eds.) Applications of Algebraic Geometry to Coding Theory, Physics and NATO Science Series II: Mathematics, Physics and Chemistry Computation, vol. 36, pp. 215-254. Kluwer, Dordrecht (2001)

16. Libgober, A.: On combinatorial invariance of the cohomology of the Milnor fiber of arrangements and the Catalan equation over function fields. In: Terao, H., Yuzvinsky, S. (eds.) Arrangements of Hyperplanes - Sapporo 2009. Advanced Studies in Pure Mathematics, vol. 62, pp. 175-187. Mathematical Society of Japan, Tokyo (2012)

17. Libgober, A., Yuzvinsky, S.: Cohomology of the Orlik-Solomon algebras and local systems. Compositio Math. 121(3), 337-361 (2000)

18. Măcinic, A.D., Papadima, Ş.: On the monodromy action on Milnor fibers of graphic arrangements. Topology Appl. 156(4), 761-774 (2009)

19. Papadima, Ş., Suciu, A.I.: The Milnor fibration of a hyperplane arrangement: from modular resonance to algebraic monodromy. Proc. London Math. Soc. 114(6), 961-1004 (2017)

20. Papini, O.: Computational Aspects of Line and Toric Arrangements. PhD thesis, Università di Pisa (2018). https://etd.adm.unipi.it/t/etd-09262018-113931/

21. Salvetti, M., Serventi, M.: Twisted cohomology of arrangements of lines and Milnor fibers. Ann. Sc. Norm. Super. Pisa Cl. Sci. (5) 17(4), 1461-1489 (2017)

22. Salvetti, M., Settepanella, S.: Combinatorial Morse theory and minimality of hyperplane arrangements. Geom. Topol. 11, 1733-1766 (2007)

23. Steenrod, N.E.: Homology with local coefficients. Ann. Math. 44(4), 610-627 (1943)

24. Suciu, A.I.: Translated Tori in the characteristic varieties of complex hyperplane arrangements. Topology Appl. 118(1-2), 209-223 (2002)

25. Suciu, A.I.: Hyperplane arrangements and Milnor fibrations. Ann. Fac. Sci. Toulouse Math. 23(2), 417-481 (2014)

26. Torielli, M., Yoshinaga, M.: Resonant bands, Aomoto complex, and real 4-nets. J. Singul. 11, 33-51 (2015)

27. Yoshinaga, M.: Milnor fibers of real line arrangements. J. Singul. 7, 220-237 (2013)

28. Yoshinaga, M.: Resonant bands and local system cohomology groups for real line arrangements. Vietnam J. Math. 42(3), 377-392 (2014)

Publisher's Note Springer Nature remains neutral with regard to jurisdictional claims in published maps and institutional affiliations. 